\title{
LEC-TMAC: Improved Energy Consumption \& Latency in TMAC Protocol for Wireless Body Area Network (WBANs)
}

\author{
Shravan Kumar Upadhayay, Mansi Gupta \\ Computer Science \& Engineering Department, Punjab Institute of Technology, Kapurthala, \\ I.K Gujral Punjab Technical University, Punjab, India \\ *Corresponding author, e-mail: shravanupadhayay@yahoo.com, mansi13gpta@yahoo.com
}

\begin{abstract}
The most common protocols for Wireless Body Area Network (WBAN) like TMAC, ZigBeeMAC, Baseline MAC protocol. In this paper, evaluating the effects of LEC-TMAC Protocol for wireless body area network basis of Latency and energy consumption. This analysis is important in point of view that the varying requirement of WBAN according to varying conditions. The proposed LEC-TMAC protocol improves the energy consumption and latency in wireless body area network by reducing the activation timeout value with the help of listen timeout. The proposed LEC-TMAC methodology is implemented using the Castalia Simulator-3.2. The results of the proposed LEC-TMAC have been compared with existing TMAC protocol and the comparison proves that the proposed approach is more effective with energy consumption and latency. As a conclusion; we invoke a number of new research challenges with regard to prospects of Wireless body area network and other issues.
\end{abstract}

Keywords: wireless body area network, WBAN, TMAC, castalia simulator, less energy consumption TMAC protocol (LEC-TMAC)

Copyright $\odot 2015$ Institute of Advanced Engineering and Science. All rights reserved.

\section{Introduction}

The Main task in wireless body area networks (WBANs) is to make the entire network/system energy efficient, for this purpose we focus on a medium access control (MAC) layer which is the most suitable level to address the energy efficiency [1-4] of the any system. Medium Access Control (MAC) layer is used to coordinate node access to the shared wireless medium. The MAC is the core of communication protocol stack which provides the basis for achieving Quality of Service (QoS) in any wireless and WBAN networks. A MAC layer should support distinct/different applications and distinct types of data such as continuous, periodic, burst and non-periodic data along with high level QoS. MAC plays a major determining factor in improving overall network performance. The fundamental task in MAC protocol is to avoid collisions and to prevent simultaneous transmissions while preserving maximum throughput, minimum latency, communication reliability and maximum energy efficiency. It is important to note that while designing MAC protocols one should keep in mind that nodes are ready to failures, constrained capabilities and barred energy resources.

MAC protocols for WBAN are analyzed in this paper to observe the effect of changing the output power of nodes. Some popular MAC protocols are TMAC, ZigBeeMAC, SMAC and Baseline MAC. TMAC and SMAC are duty- cycling protocols Where SMAC is the previous version of TMAC. ZigBeeMAC protocol can use CSMA/CA or TDMA schemes and gives better energy efficiency and throughput in simple WBAN having less number of nodes. Baseline MAC protocol engage CSMA/CA methodology and its performance is average in WBAN.

WBASN can be applied to different scenarios such as monitoring of an individual patient from home, monitoring of few patients from intensive care units (ICU) and monitoring of many patients from hospital wards. Generally in these scenarios there is no requirement for large network; single-hop communications with star or clustered-based topologies are most popular in WBASN $[5,6]$. In this context, energy consumption and latency are two important design constraints along with the miniature size of the sensor nodes. The nodes that are implanted inside the body require high energy efficiency, in other words the lifetime of the sensor nodes 
and network needs to be prolonged by the use of ultra-low power technologies and protocols [3, 7].

In a typical WSN platform, the radio transceiver consumes most of the power. Radio activity is controlled by the medium access control (MAC) layer, therefore it is necessary to design an ultra low power and energy efficient MAC protocol suitable for WBASN(wireless body area sensor network).

In this paper, we have provided a Performance Analysis of Mac Protocols for WBAN Based on Application Packet Rate and compare the results bases of different parameters. More specifically, in this paper we focus on Performance analysis of T-MAC and LEC-TMAC protocol based on total energy consumption and application level latency for WBAN.

\section{Related Work}

Several researchers are being attracted towards analysis of performances of MAC protocols in WBAN. In a study of performance of MAC protocols in WBAN several protocols are tested for their compatibility with WBAN. This study suggests that T-MAC [7, 9], S-MAC, ZigBeeMAC [3], and Baseline MAC [5] protocols perform efficiently in case of WBAN [1]. In another research work author analyzed MAC protocols to suggest the most energy efficient protocol for WBAN [3], [5-6], [8]. In some research works T-MAC protocol is analyzed for its better performance than other protocols [7].

\section{Research Method}

For analysis of 6 nodes architecture of WBAN is taken which are numbered from 0 to 5 . These nodes are arranged in star topology and all the nodes from 1 to 5 are connected to node 0 which is working as network coordinator. Node 0 is taken as sink in WBAN architecture for isolating it for proper analysis.

Latency and energy consumption are most important parameters for evaluating the performance of WBAN. Latency in receiving packets is also an essential parameter in performance of a MAC protocol. These parameters are analyzed at Random Application Packets rate for various MAC protocols and results are compared to present the effect of change in latency \& energy consumptions of nodes. Simulation and setting parameters are shown in Table 1 and Table 2.

Table 1. Simulation Parameters

\begin{tabular}{lc}
\hline Simulation Parameters & Default Values \\
\hline Number of nodes & 6 \\
Physical Data Rate & $1024 \mathrm{kbps}$ \\
Phy overhead & 6 \\
Rx sensitivity & Default \\
Tx Transmission Power & Default(-15db) \\
\hline
\end{tabular}

Table 2. Setting Parameters

\begin{tabular}{lc}
\hline \multicolumn{1}{c}{ Parameter Name } & Parameters \\
\hline Channel type & Channel/ wireless \\
Radio Propagation & Propagation/Two Ray Ground \\
Topology & Star Topology \\
Link Layer type & MAC Layer \\
Network Interface Type & Phy/Wireless Phy \\
MAC type & 802.15 .4 BAN \\
No of mobile nodes & 06 \\
Contention Period & Default \\
listenTimeout & Default \\
conservativeTA & Default \\
MAC Protocol & TMAC \\
\hline
\end{tabular}

\section{MAC Protocols for Wireless Body Area Network}

MAC protocols used in WBAN must be low energy consuming, accurate and with less latency. The most important thing is the protocol should give good performance on varying traffic 
load.

TMAC: Time-out MAC[9],TMAC is a popular MAC for WBAN as it employs many techniques to keep the energy consumption low and trying to keep performance high by adapting its duty cycle according to the traffic needs. It uses flexible duty cycles for rising energy efficiency. In T-MAC, node uses the concept of sleep and wakeup, the node wakes up after time slot assignment, send pending messages. If there is no activation event for Time Interval (TA), the node goes back to sleep mode again. If a node sends Route To Send (RTS) and does not receive Clear To Send (CTS), then sends RTS two more times before going to sleep. To solve initial sleep problem, it uses imminent RTS for taking priority on full buffer.

Some important parameters for WBAN

1. Frame Time

2. listen Timeout

3. Contention Periods

4. Conservative TA

5. Collision Resolutions

6. allow sink sync

7. Wait Timeout and etc.

load.

Advantage: delay is minimized. It also exceeds other MAC protocols under variable

Disadvantage: sleeping problems.

5. Proposed Less Energy Consumption (LEC-TMAC) Algorithm

Algo: Extend Active Period Values Varying With the Listen Timeout

Step 1: \#Define simtime_t curTime = getClock(), Define ListenTimeout ,Activation Timeout and Extra

Step 2: Then Check conditions

1) if (conservativeTA) Then set CurTime Value as

CurTime += extra;

Otherwise check while (activationTimeout < curTime) condition

if given condition is true then the $A T=L T / 2$;

2) if (curTime + listenTimeout < activationTimeout) then return Default value and set Activation Timeout value is Half of the Listen Timeout Value.

$A T+=L T / 2$

3) else if (activationTimeout < curTime + listenTimeout + extra)

Then the ActivationTimeout is Sum of the curTime + listenTimeout /2+ extra

Step3: Set The Timer Value and Check the TA.

\section{Performance Evaluations and Results}

For simulation setup, we consider 6 nodes firmly fixed on a human body. The nodes are connected to the coordinator in a star topology and generate up-link traffic only. The simulator used in this paper is the open source Castalia 3.2[23]. which is integrated under the OMNET++ simulator. All nodes send packets to the hub, which is the node number zero.

The performance of various MAC protocols is calculated by considering the following performance parameters:

1. Energy Consumption

2. Latency

\subsection{Total Energy Consumption}

It is Obvious that send packets from nodes to sink nodes, then energy consumption of the WBAN will effected up to a great extent.

The total energy consumption is represented by the total transmission and reception energy of all wireless nodes. The energy the radio dissipates to run the circuitry for the transmitter and receiver are denoted by ETXelec and ERXelec.

Total Energy Consumption $=$ ETXelec + ERXelec

ETXelec $=$ total transmission energy

$\mathrm{ERXelec}=$ total reception energy

TELKOMNIKA Vol. 15, No. 3, September 2015: $430-435$ 


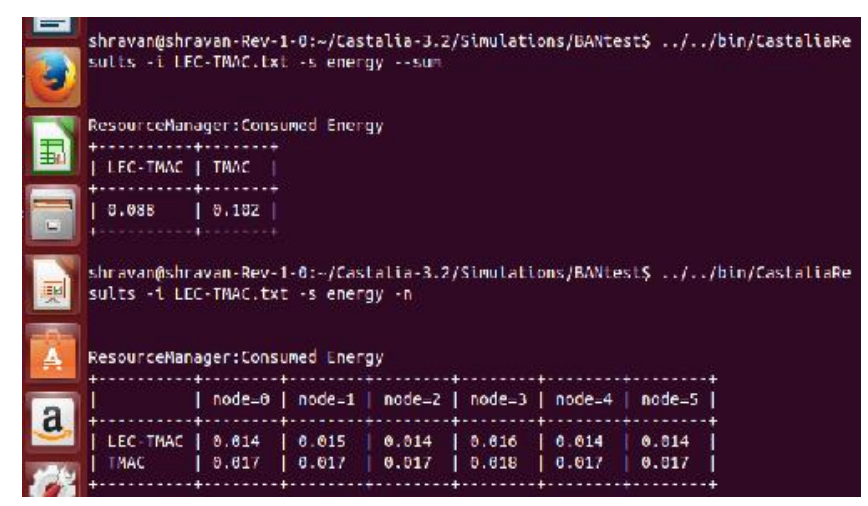

Figure 1. Simulation result based on energy consumption

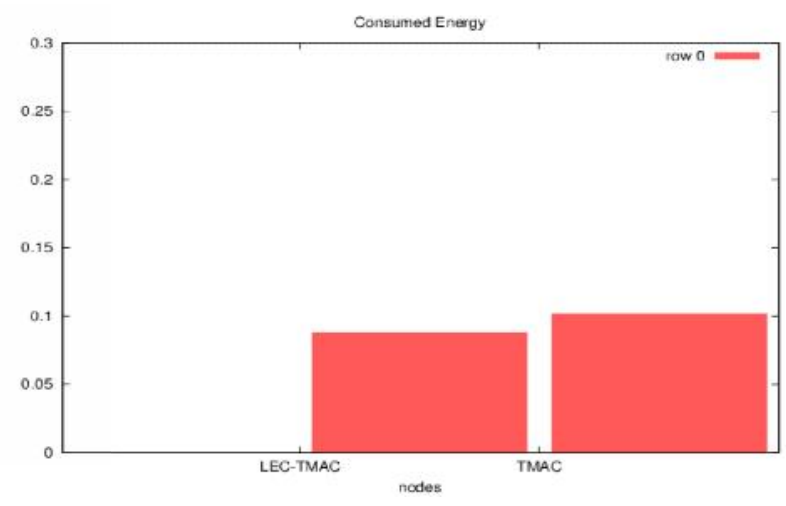

Figure 2. Total Energy consumed

Figure 2, this fact is also clear in this graph that the Total energy consumption of LECTMAC is less than as Compare to T-MAC.

\subsection{Latency Analysis}

The latency time is equal to the arrived message time minus the message creation time.

\section{LT $=$ AMT $-\mathbf{M C T}$}

Where $\mathrm{LT}=$ latency time, $\mathrm{AMT}=$ Arrived Message time, $\mathrm{MCT}=$ Message Creation Time

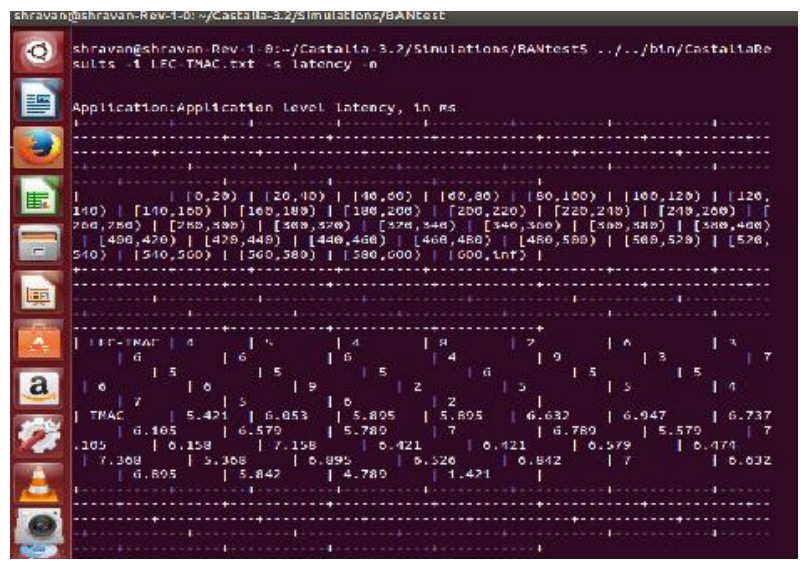

Figure 3. Simulation result based on application level Latency 


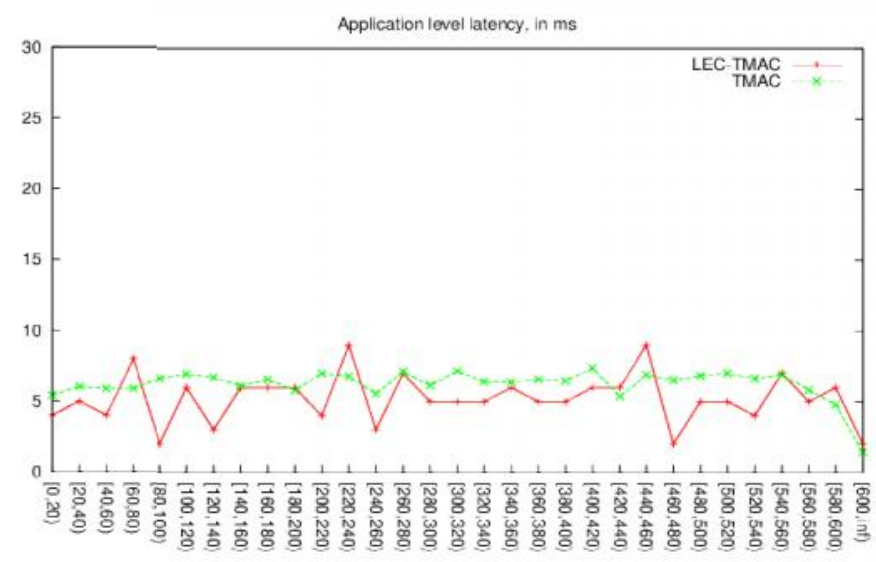

Figure 4. Application level Latency

Figure 4 in this graph it is clear that the application level latency of per nodes is different in TMAC. And the Simulation result clearly shows that per node application level Latency of LEC-TMAC is better than the TMAC Protocols

\section{Conclusion and Future Work}

The simulation result shows that TMAC protocol performs better than the other existing MAC protocols such as ZigBeeMAC and BaselineMAC. But still sleeping problem remain exist in TMAC which cause loss of energy and power consumptions.

The proposed LEC-TMAC protocol improves the energy consumption and latency in wireless body area network by reducing the activation timeout value and extend Active period of the sensor. The proposed LEC-TMAC methodology is implemented using the Castalia Simulator-3.2. The results of the proposed LEC-TMAC have been compared with existing TMAC protocol and the comparison proves that the proposed approach is more effective with energy consumption and latency.

Furthermore, the hardware implementation of LEC-TAMC can be done by varying different parameters and observing the results. The proposed work does not include throughput, PDR and network lifetime in the WBAN structure. In Future works we can improve the throughput, Packets Delivery Ratio and many other factors in LEC-TMAC Protocol.

\section{References}

[1] Ullah S, Shen B, Riazul Islam S, Khan P, Saleem S, Sup Kwak K. A Study of MAC Protocols for WBANs. Sensors. 2010; 10(1): 128-145.

[2] I C, Li HB, Kohno R. Performance evaluation of IEEE 802.15.4 for wireless body area network. In Proceedings of IEEE International Conference on ICC Workshops. Dresden, Germany. 2009: 1-5.

[3] Fang G, Dutkiewicz E. BodyMAC: Energy efficient TDMA-based MAC protocol for wireless body area networks. In Proceedings of IEEE ISCIT 2009. Incheon, Korea. 2009.

[4] Kohvakka M, Suhonen J, Hamalainen TD, Hannikainen M. Energy-efficient reservation- basedmedium access control protocol for wireless sensor networks. EURASIP J, Wireless Communication Networks. $2010 ; 22$.

[5] Niamat Ullah, Pervez Khan, Kyung Sup Kwak. A Very Low Power MAC (VLPM) Protocol for Wireless Body Area Networks. Sensors. 2011; 11: 3717-3737.

[6] Timmons NF, Scanlon WG. Analysis of the performance of IEEE 802.15.4 for medical sensor body area networking. In Proceedings of First Annual IEEE Communications Society, Sept. 2009 Conference on Sensor and Ad Hoc Communications and Networks. Santa Clara, CA, USA. 2004: 4-7.

[7] Van Dam T, Langendoen K. An adaptive energy efficient MAC protocol for WSNs. In Proceedings of ACM Conf. on Embedded Networked Sensor Systems, SenSys 2003. Los Angeles, CA, USA. 2003: 171-180.

[8] Zhen B, Li HB, Kohno R. IEEE body area networks for medical applications. In Proceedings of IEEE International Symposium on Wireless Communication. 
[9] Khan NP, Boncelet C. PMAC: Energy efficient medium access control protocol for wireless sensor networks. In Proceedings of IEEE Military Communications Conference. Washington, DC, USA. 2006: 1-5, 23-25.

[10] Rajendran V, Garcia Luna Aveces JJ, Obraczka K. Energy-efficient, application-aware medium access for sensor networks. In Proceedings of IEEE Mobile Adhoc and Sensor Systems Conference. Washington, DC, USA. 2005: 8630.

[11] Fatehy M, Kohno R. Body Area Networks performance analysis using UWB. Engineering in Medicine and Biology Society (EMBC), 2013 35th Annual International Conference of the IEEE. 2013: 12181221.

[12] El azhari M, Toumanari A, Latif R. Performance analysis of IEEE 802.15.6 and IEEE 802.15.4 for wireless body sensor networks. Multimedia Computing and Systems (ICMCS), 2014 International Conference. 2014: 910-915.

[13] Singh P, Varma S. An improved TMAC protocol for Wireless Sensor Networks. Signal Propagation and Computer Technology (ICSPCT), 2014 International Conference. 2014.

[14] Smriti joshi, Anant Kr Jaiswal, Pushpendra Kr Tyagi A Novel Analysis of T Mac and S Mac Protocol for Wireless Sensor Networks Using Castalia. International Journal of Soft Computing and Engineering (IJSCE). 2013; 2(6). 